\title{
Evaluation of Some Barley Landraces
}

\author{
Amer M. Abdel Aziz and Khamis I. Saad ${ }^{1}$
}

\begin{abstract}
This work was carried out on 18 genotypes of barely (16 out of them are landraces and two are commercial cultivars). These genotypes were cultivated at the farm of Nubaria Agricultural Research Station for the two successive seasons of 2012/2013 and 2013/2014 and evolutional of some characters were significant differ between genotypes with respect of the green yield character.

The data showed that the commercial varieties are higher than that of the landraces. Plant height $(\mathrm{cm})$ showed that genotype 1 and 11 were higher than that of the commercial varieties in both seasons. Also the genotype 12 was found to be higher. No. of days to heading (days) in one season: Genotypes 2, 11 and 13 were proven to be higher compared with the commercial varieties. But in the second season genotypes 11 and 13 were higher compared with that of the commercial varieties. Grain yield $\left(\mathrm{g} \mathrm{m}^{-2}\right)$ in one season: No. $3 \& 16$ were found to be higher landraces genotypes compared with the commercial cultivars but its less than commercial cultivars. But in the second season were genotypes 3 , 5and 13 higher compared with the remaining landraces but it's lower than the commercial the commercial varieties. Spike length $(\mathrm{cm})$ was genotype 2 hieher compared with landraces genotypes in both seasons. No. plants per $\mathrm{m}^{2}$ in one season: Genotype 4 and 5 were proven to be higher compared with that of the commercial varieties. But in the second season were 5 and 7 higher compared with that of landraces genotypes. Weight of 1000 grains (g) in one season: No. 1 and 4 proved to be higher compared with that of commercial ones. But in the second season were 1 and 4 hieher compared with landraces genotypes. Correlation between no. of days to heading (days) was significant with spike length $(\mathrm{cm})$ and negative significant with grain yield but 1000 grains (g) during the first season of 2012/2013, but in the second season 2013/2014 it was significant with spike length $(\mathrm{cm})$ and negative high significant with 1000 grains.
\end{abstract}

Key words: Land races, Barley, Yield and Yield components.

\section{INTRODUCTION}

Barley (Hordeum vulgare L.) is one of the most ancient crops among the cereals and has played a significant role in the development of agriculture (Ullrich, 2011). Today, cultivated barley is grown in much more diverse ecogeographic environmental conditions as compared to other crop species. It can be planted from the tropics to marginal areas in north and high altitudes (Nevo 1992). Before the $20^{\text {th }}$ century barley was mainly used as a human food but presently it is used mainly as animal feed. It is also used for malt production and human consumption. Barley, in comparison with other cereal crops has a better fodder value including both grain and straw. In most of developed countries barley straw is used for animal bedding, whereas, in the developing counties it is also used for animal feed (Akar et al., 2012).

Recently, methods for the estimation of grain yield or quality, based on spectral characteristics of the barley stand in various growth stages have been developed (Wessteiner and Kühbauch 2005, Zhao et al. 2005).

The present study was undertaken to:

(a) Evaluation of plant height $(\mathrm{cm})$, spike length $(\mathrm{cm})$, grain yield $\left(\mathrm{g} \mathrm{m}^{-2}\right)$, no. of days to heading (days), no. plants in $\mathrm{m}^{-2}$ and weight of 1000 grains (g) and (b) Correlation between characters under studies during the successive seasons of 2012/2013 and 2013/201

\section{MATERIALS AND METHODS}

\section{Plant materials:}

Barley genoypes (16 landraces) and two commercial cultivars namely (Giza 2000 and Giza131) have been evaluated under normal conditions.

Experiments farm were carried out during two successive seasons of 2012/2013 and 2013/2014 at the Farm of Nubaria, Agric. Res. Center, Ministry of Agric, Egypt. (Table, 1)

Barley (Hordeum vulgar L.) sowing dates were December $15^{\text {th }}$ and $17^{\text {th }}$ in the first and second seasons, respectivelly. While, harvesting was in May $15^{\text {th }}$ and $20^{\text {th }}$ at the two successive seasons. The experiment was carried out in a randomized complete block design (RCBD) with 3 replicates. A plot size of $3 \times 1.2 \mathrm{~m}$ having 6 rows was used with seeding rate of $120 \mathrm{~kg} \mathrm{ha}^{-}$ 1. Basal doses of $108 \mathrm{~kg}$ nitrogen, $36 \mathrm{~kg}$ phosphorous and $58 \mathrm{~kg}$ Potassium ha ${ }^{-1}$ were applied in the time of sowing. Grain yield was obtained for each genptype, which was later converted to yield per square meter.

Measured characters were included; plant height (stem and spike), length of the spike, grain yield $\left(\mathrm{gm} \mathrm{m}^{-2}\right)$, number of the days to heading $(50 \%$ spikes on plant), number of plants per $\mathrm{m}^{-2}$ and weight of 1000 grains (gm).

\footnotetext{
${ }^{1}$ Genetic Resources Research Dept. in Field crops research institute, ARC, Giza, Egypt.

Received December 3 , 2015, Accepted December 28, 2015
} 
Table 1. Barley genotypes included in the study

\begin{tabular}{cccccc}
\hline Genotypes & Type & Chromosome Number & Genotypes & Type & Chromosome Number \\
\hline 7543 & 6 rows & 14 & 7466 & 6 & 14 \\
\hline 7436 & 2 rows & 14 & 7471 & 2 & 14 \\
\hline 7440 & 6 rows & 14 & 7476 & 6 & 14 \\
\hline 7441 & 2 rows & 14 & 7479 & 2 & 14 \\
\hline 7442 & 6 rows & 14 & 7483 & 2 & 14 \\
\hline 7450 & 2 rows & 14 & 7458 & 6 & 14 \\
\hline 7452 & 2 rows & 14 & 7489 & 6 & 14 \\
\hline 7454 & 2 rows & 14 & Giza 131 & 6 & 14 \\
\hline 7485 & 6 rows & 14 & Giza 2000 & 6 & \\
\hline
\end{tabular}

\section{Soil initial state analysis:}

Soil analysis of the soil samples (table 2) indicates that the surface soil layer $(0-30 \mathrm{~cm})$ has a light texture of sandy loam with a high content of $\mathrm{CaCO}_{3} \%(22.45$ and $25.11 \%$ for the two seasons, respectively). Soils of the both seasons were alkaline and non-saline with low available NPK and low content of O.M. \% indicating their deficient fertility status.

\section{Statistical analysis}

Data were subjected to the analysis of variance ANOVA using (LSD) Test following the randomized complete block design (RCBD), with three replications for each treatment. The least significant differences (L.S.D) at the $0.05 \leq$ level were determined according to computer program (COSTAT software, 1988)

\section{RESULTS AND DISCUSSION}

\section{Plant height (cm):}

Was calculated and data are given in table (5 and 6). Means ranged from 66 to $94 \mathrm{~cm}$ for the genotypes 7485 and 7442 to the genotype 7476 , respectively, at the first season, and ranged from 66 to $94 \mathrm{~cm}$ for the genotype 7442 to the genotype 7476 , respectively, at the second season.

\section{Number of days to heading (days):}

The data are given in table (5 and 6). Means ranged from 72.33 to 85 days for the genotype 7440 to the genotype 7471 , respectively, at the first season, and ranged from 71.33 to 85.67 days for the genotype 7440 to the genotype 7471 , respectively, at the second season.

\section{Grain yield $\left(\mathrm{gm}^{-2}\right)$ :}

It was calculated and data are given in table ( 7 and 8). Means ranged from 214.67 to $432 \mathrm{gm}^{-2}$ for the genotype 7454 to cultivate Giza 2000, respectively, at the first season, and ranged from 244.67 to $443.67 \mathrm{gm}^{-2}$ for the genotype 7454 to cultivate Giza 2000, respectively, at the second season.

\section{Spike length (cm):}

The data are given in table ( 7 and 8 ). Means ranged from 5.4 to $9.83 \mathrm{~cm}$ for the genotype 7440 to cultivate Giza 2000, respectively, at the first season, and ranged from 5.57 to $9.97 \mathrm{~cm}$ for the genotype 7440 to cultivate Giza 2000, respectively, at the second season.

Number of plant per $\mathbf{m}^{2}$ :

The data are given in table ( 7 and 8 ). Means ranged from 397.67 to 1080 for the genotype 7489 to the genotype 7450 , respectively, at the first season, and ranged from 401 to 1099.3 for the genotype 7489 to the genotype 7450 , respectively, at the second season.

Table 2. Some initial soil physicochemical characteristics of the surface layer $(0-30 \mathrm{~cm})$ of the experimental seasons 2012/2013 and 2013/2014

\begin{tabular}{lcc}
\hline \multicolumn{1}{c}{ Soil characteristics } & \multicolumn{2}{c}{ Experimental season } \\
\cline { 2 - 3 } & $\mathbf{2 0 1 2 / 2 0 1 3}$ & $\mathbf{2 0 1 3 / 2 0 1 4}$ \\
\hline Soil EC, $\mathrm{dS} \mathrm{m}^{-1}$ & 2.17 & 2.43 \\
Soil $\mathrm{pH}(1: 2.5)$ & 8.27 & 8.24 \\
Total $\mathrm{CaCO}_{3} \%$ & 22.45 & 25.11 \\
O.M $\%$ & 0.27 & 0.31 \\
Soil texture & Sandy Loam & Sandy Loam \\
Available macronutrients ppm & & \\
$\mathrm{N}$ & 41.62 & 39.73 \\
$\mathrm{P}$ & 3.16 & 3.28 \\
$\mathrm{~K}$ & 89.74 & 90.62 \\
\hline
\end{tabular}


Table 3. Mean monthly climatic data for 2012-2013 growing seasons in Nubaria expermintal station

\begin{tabular}{lcccc}
\hline \multirow{2}{*}{ Month } & \multicolumn{2}{c}{ Temperature $\left({ }^{\circ} \mathbf{C}\right)$} & \multirow{2}{*}{ Rainfall (mm) } & \multirow{2}{*}{ Relative Humidity (\%) } \\
\cline { 2 - 3 } & $\mathbf{m a x}$ & $\mathbf{m i n}$ & 0.27 & 59.49 \\
\hline November 2012 & 26.01 & 16.62 & 1.35 & 59.36 \\
\hline December 2012 & 19.92 & 10.90 & 1.97 & 61.63 \\
\hline January 2013 & 18.13 & 6.72 & 0.47 & 51.35 \\
\hline February 2013 & 20.88 & 7.64 & 0.10 & 43.27 \\
\hline April 2013 & 25.43 & 10.65 & 0.12 & 47.54 \\
\hline May 2013 & 27.34 & 12.16 & 0.10 & 41.95 \\
\hline
\end{tabular}

Table 4. Mean of monthly climatic data for 2013-2014 growing seasons in Nubaria expermintal station

\begin{tabular}{lcccc}
\hline \multirow{2}{*}{ Month } & \multicolumn{2}{c}{ Temperature $\left({ }^{\circ} \mathbf{C}\right)$} & \multirow{2}{*}{ Rainfall $(\mathbf{m m})$} & \multirow{2}{*}{ Relative Humidity (\%) } \\
\cline { 2 - 3 } & $\mathbf{m a x}$ & $\mathbf{m i n}$ & 0.26 & 61.12 \\
\hline November 2013 & 25.02 & 14.38 & 0.63 & 61.65 \\
\hline December 2013 & 18.85 & 9.14 & 0.64 & 64.01 \\
\hline January 2014 & 19.23 & 8.64 & 0.47 & 62.71 \\
\hline February 2014 & 20.62 & 8.25 & 0.36 & 49.94 \\
\hline April 2014 & 23.88 & 10.27 & 0.23 & 45.22 \\
\hline May 2014 & 28.22 & 12.99 & 0.19 & 42.19 \\
\hline
\end{tabular}

Table 5. Means $\left(x^{-} \pm S\right.$. d) of plant characters under study during the first season of 20122013

\begin{tabular}{|c|c|c|c|c|c|}
\hline \multirow{3}{*}{ No. } & \multirow{3}{*}{ Genotypes } & \multicolumn{4}{|c|}{ characters } \\
\hline & & \multicolumn{4}{|c|}{ Number of days to heading (days) } \\
\hline & & $\mathbf{x}^{-}$ & S. d & & S. d \\
\hline 1 & 7543 & $90.33 \mathrm{AC}$ & 3.79 & 7543 & 2.00 \\
\hline 2 & 7436 & $76.33 \mathrm{GF}$ & 5.51 & 7436 & 2.00 \\
\hline 3 & 7440 & $76.33 \mathrm{GF}$ & 2.52 & 7440 & 0.58 \\
\hline 4 & 7441 & $78.33 \mathrm{DF}$ & 3.21 & 7441 & 1.15 \\
\hline 5 & 7442 & $66.00 \mathrm{H}$ & 2.65 & 7442 & 1.00 \\
\hline 6 & 7450 & $74.33 \mathrm{FH}$ & 5.86 & 7450 & 1.15 \\
\hline 7 & 7452 & $77.33 \mathrm{GF}$ & 9.02 & 7452 & 3.61 \\
\hline 8 & 7454 & 79.33GDF & 4.51 & 7454 & 1.52 \\
\hline 9 & 7485 & $66.00 \mathrm{H}$ & 9.54 & 7485 & 0.58 \\
\hline 10 & 7466 & $71.00 \mathrm{GH}$ & 8.00 & 7466 & 0.58 \\
\hline 11 & 7471 & $92.33 \mathrm{AB}$ & 4.93 & 7471 & 1.00 \\
\hline 12 & 7476 & $94.00 \mathrm{~A}$ & 4.00 & 7476 & 0.58 \\
\hline 13 & 7479 & $76.00 \mathrm{EFG}$ & 5.29 & 7479 & 0.58 \\
\hline 14 & 7483 & $76.00 \mathrm{EFG}$ & 3.61 & 7483 & 1.53 \\
\hline 15 & 7458 & $87.33 \mathrm{ABCD}$ & 4.04 & 7458 & 1.15 \\
\hline 16 & 7489 & 81.00CDEF & 7.21 & 7489 & 0.58 \\
\hline 17 & Giza 131 & 85.00ABCDE & 4.36 & Giza 131 & 1.00 \\
\hline 18 & Giza 2000 & 83.67BCDEF & 6.03 & Giza 2000 & 1.00 \\
\hline
\end{tabular}


Table 6. Means $\left(x^{-} \pm S . d\right)$ of plant characters under study during the second season of 20132014

\begin{tabular}{cccccc}
\hline \multirow{2}{*}{ No. } & \multirow{2}{*}{ Genotypes } & \multicolumn{4}{c}{ Characters } \\
\cline { 3 - 6 } & & \multicolumn{2}{c}{ Plant height (cm) } & \multicolumn{2}{c}{ Number of days to heading (days) } \\
\cline { 2 - 6 } & \multicolumn{2}{c}{$\mathbf{x}^{-}$} & & $\mathbf{x}^{-}$ & $\mathbf{S}$. d \\
\hline 1 & 7543 & $93.67 \mathrm{~A}$ & 1 & $75.00 \mathrm{IH}$ & 1.00 \\
\hline 2 & 7436 & $79.67 \mathrm{CD}$ & 2 & $80.00 \mathrm{BCD}$ & 2.00 \\
\hline 3 & 7440 & $77.67 \mathrm{CD}$ & 3 & $71.33 \mathrm{~K}$ & 1.15 \\
\hline 4 & 7441 & $79.00 \mathrm{CD}$ & 4 & $76.67 \mathrm{FGH}$ & 0.58 \\
\hline 5 & 7442 & $66.00 \mathrm{E}$ & 5 & $72.33 \mathrm{JK}$ & 1.15 \\
\hline 6 & 7450 & $75.33 \mathrm{D}$ & 6 & $74.33 \mathrm{IJ}$ & 0.57 \\
\hline 7 & 7452 & $75.33 \mathrm{D}$ & 7 & $78.33 \mathrm{DEF}$ & 3.79 \\
\hline 8 & 7454 & $80.67 \mathrm{BCD}$ & 8 & $76.00 \mathrm{GHI}$ & 1.00 \\
\hline 9 & 7485 & $67.00 \mathrm{E}$ & 9 & $72.00 \mathrm{~K}$ & 1.00 \\
\hline 10 & 7466 & $74.67 \mathrm{D}$ & 10 & $80.67 \mathrm{BC}$ & 1.15 \\
\hline 11 & 7471 & $88.00 \mathrm{AB}$ & 11 & $85.67 \mathrm{~A}$ & 0.58 \\
\hline 12 & 7476 & $94.00 \mathrm{~A}$ & 12 & $77.33 \mathrm{FG}$ & 0.58 \\
\hline 13 & 7479 & $75.33 \mathrm{D}$ & 13 & $82.00 \mathrm{~B}$ & 1.00 \\
\hline 14 & 7483 & $76.33 \mathrm{D}$ & 14 & $79.67 \mathrm{CDE}$ & 0.58 \\
\hline 15 & 7458 & $84.00 \mathrm{BC}$ & 15 & $77.00 \mathrm{FGH}$ & 1.00 \\
\hline 16 & 7489 & $78.67 \mathrm{CD}$ & 16 & $76.67 \mathrm{FGH}$ & 1.15 \\
\hline 17 & Giza 131 & $84.00 \mathrm{BC}$ & 17 & $77.67 \mathrm{EFG}$ & 1.53 \\
\hline 18 & Giza 2000 & $85.00 \mathrm{BC}$ & 18 & $72.00 \mathrm{~K}$ & 1.00 \\
\hline
\end{tabular}

Table 7. Means $\left(x^{-}+\right.$S. d) of yield and yield components characters under study during the first season of 2012-2013

characters

\begin{tabular}{|c|c|c|c|c|c|c|c|c|c|}
\hline \multirow[t]{2}{*}{ No. } & \multirow[t]{2}{*}{ Genotypes } & \multicolumn{2}{|c|}{ Grain yield (gm-2) } & \multicolumn{2}{|c|}{ Spike length $(\mathrm{cm})$} & \multicolumn{2}{|c|}{ Number of plant per m2 } & \multicolumn{2}{|c|}{ Weight of 1000 (grams) } \\
\hline & & $\mathbf{x}^{-}$ & S. d & $\mathbf{x}^{-}$ & S. d & $\mathbf{x}^{-}$ & S. d & $\mathbf{x}^{-}$ & S. d \\
\hline 1 & 7543 & 303.67CDEF & 12.58 & 7.83BCD & 0.76 & $483.33 \mathrm{GH}$ & 32.14 & $56.20 \mathrm{AB}$ & 0.90 \\
\hline 2 & 7436 & 306.00CDEF & 23.58 & $9.17 \mathrm{AB}$ & 1.04 & $560.00 \mathrm{GH}$ & 70.00 & 51.33BCDE & 0.68 \\
\hline 3 & 7440 & $357.67 \mathrm{BC}$ & 63.00 & $5.40 \mathrm{~F}$ & 0.52 & $596.67 \mathrm{FGH}$ & 50.33 & 51.13BCDEF & 3.90 \\
\hline 4 & 7441 & $222.33 \mathrm{GH}$ & 10.79 & $7.17 \mathrm{CDE}$ & 0.86 & $831.00 \mathrm{BCD}$ & 97.08 & $52.83 \mathrm{ABCD}$ & 1.40 \\
\hline 5 & 7442 & 333.00BCDE & 91.16 & 6.47DEF & 1.011 & $870.00 \mathrm{~B}$ & 78.10 & $50.83 \mathrm{CDEF}$ & 0.49 \\
\hline 6 & 7450 & 285.33DEFG & 9.07 & $7.73 \mathrm{BCD}$ & 1.41 & $1080.0 \mathrm{~A}$ & 270.74 & 45.93FGHI & 1.76 \\
\hline 7 & 7452 & $260.00 \mathrm{FGH}$ & 47.95 & $8.53 \mathrm{ABC}$ & 1.07 & $839.00 \mathrm{BC}$ & 121.08 & $45.47 \mathrm{GHIJ}$ & 2.45 \\
\hline 8 & 7454 & $214.67 \mathrm{H}$ & 15.82 & $6.10 \mathrm{EF}$ & 0.90 & 632.00DEFG & 72.81 & 47.63DEFGH & 5.12 \\
\hline 9 & 7485 & $254.33 \mathrm{FGH}$ & 39.72 & $5.43 \mathrm{~F}$ & 0.59 & 648.67CDEFG & 60.67 & $43.23 \mathrm{HIJ}$ & 1.26 \\
\hline 10 & 7466 & 323.33CDEF & 54.28 & 6.70DEF & 0.52 & $458.00 \mathrm{GH}$ & 60.65 & $42.07 \mathrm{IJ}$ & 3.10 \\
\hline 11 & 7471 & $258.00 \mathrm{FGH}$ & 31.76 & $8.50 \mathrm{ABC}$ & 0.87 & 578.33FGH & 109.00 & $43.03 \mathrm{HIJ}$ & 3.15 \\
\hline 12 & 7476 & $358.33 \mathrm{BC}$ & 50.86 & $6.10 \mathrm{EF}$ & 0.90 & $624.00 \mathrm{EFG}$ & 115.53 & $40.57 \mathrm{~J}$ & 2.86 \\
\hline 13 & 7479 & $270.00 \mathrm{EFGH}$ & 20.07 & 6.73DEF & 1.10 & $814.33 \mathrm{BCDE}$ & 72.28 & 47.90DEFH & 3.91 \\
\hline 14 & 7483 & $278.00 \mathrm{EFGH}$ & 14.73 & $8.87 \mathrm{AB}$ & 1.27 & 807.00BCDE & 87.13 & 45.13GHIJ & 4.03 \\
\hline 15 & 7458 & $355.00 \mathrm{BCD}$ & 46.13 & 6.53DEF & 0.45 & 776.33BCDEF & 123.03 & 49.40DEFG & 3.87 \\
\hline 16 & 7489 & $362.00 \mathrm{BC}$ & 44.79 & $5.60 \mathrm{~F}$ & 0.60 & $397.67 \mathrm{H}$ & 86.57 & 47.40EFGH & 2.49 \\
\hline 17 & Giza 131 & $401.00 \mathrm{AB}$ & 9.00 & $9.10 \mathrm{AB}$ & 0.90 & $895.00 \mathrm{AB}$ & 127.08 & $55.50 \mathrm{ABC}$ & 4.81 \\
\hline 18 & Giza 2000 & $432.00 \mathrm{~A}$ & 48.59 & $9.83 \mathrm{~A}$ & 0.64 & $973.33 \mathrm{~A}$ & 265.01 & $57.43 \mathrm{~A}$ & 4.70 \\
\hline
\end{tabular}


Table 8. Means $\left(x^{-} \pm\right.$S. $\left.d\right)$ of yield and yield components characters under study during the second season of 2013-2014

\begin{tabular}{|c|c|c|c|c|c|c|c|c|c|}
\hline \multirow{3}{*}{ No. } & \multirow{3}{*}{ Genotypes } & \multicolumn{8}{|c|}{ Characters } \\
\hline & & \multicolumn{2}{|c|}{ Grain yield (gm-2) } & \multicolumn{2}{|c|}{ Spike length $(\mathrm{cm})$} & \multicolumn{2}{|c|}{ Number of plant per $\mathrm{m} 2$} & \multicolumn{2}{|c|}{ Weight of 1000 (grams) } \\
\hline & & $\mathbf{x}^{-}$ & S. d & $\mathbf{x}^{-}$ & S. d & $\mathbf{x}^{-}$ & S. d & $\mathbf{x}^{-}$ & S. d \\
\hline 1 & 7543 & $311.00 \mathrm{EF}$ & 9.54 & $8.23 \mathrm{C}$ & 0.32 & $462.00 \mathrm{GH}$ & 14.80 & $55.87 \mathrm{AB}$ & 2.18 \\
\hline 2 & 7436 & 318.00DEF & 20.22 & $9.27 \mathrm{AB}$ & 0.55 & $577.00 \mathrm{EFG}$ & 86.75 & $51.73 \mathrm{BCD}$ & 0.81 \\
\hline 3 & 7440 & 353.33BCDE & 47.37 & $5.57 \mathrm{G}$ & 0.30 & $615.67 \mathrm{EF}$ & 20.40 & $51.77 \mathrm{BCD}$ & 2.31 \\
\hline 4 & 7441 & $245.00 \mathrm{G}$ & 44.98 & $7.33 \mathrm{D}$ & 0.45 & $857.00 \mathrm{CD}$ & 64.65 & $52.67 \mathrm{BC}$ & 3.09 \\
\hline 5 & 7442 & 353.67BCDE & 34.59 & 6.93DE & 0.72 & 897.00BCD & 16.09 & 51.33BCDE & 1.78 \\
\hline 6 & 7450 & $320.00 \mathrm{DEF}$ & 55.38 & $8.27 \mathrm{C}$ & 1.07 & $1099.3 \mathrm{~A}$ & 175.54 & $45.27 \mathrm{GH}$ & 4.35 \\
\hline 7 & 7452 & $294.00 \mathrm{FG}$ & 7.00 & $8.60 \mathrm{BC}$ & 0.66 & $875.33 \mathrm{BCD}$ & 106.27 & 47.17DEFG & 3.45 \\
\hline 8 & 7454 & $244.67 \mathrm{G}$ & 8.08 & 6.57DEF & 0.70 & $634.00 \mathrm{E}$ & 35.93 & $46.10 \mathrm{FGH}$ & 4.54 \\
\hline 9 & 7485 & $303.67 \mathrm{EF}$ & 43.78 & $5.80 \mathrm{FG}$ & 0.44 & $643.67 \mathrm{E}$ & 54.05 & $43.90 \mathrm{GH}$ & 2.35 \\
\hline 10 & 7466 & $349.67 \mathrm{CDE}$ & 34.79 & $6.97 \mathrm{D}$ & 0.21 & 497.00FGH & 61.65 & $41.90 \mathrm{H}$ & 2.86 \\
\hline 11 & 7471 & $294.33 \mathrm{FG}$ & 12.58 & $8.73 \mathrm{BC}$ & 0.47 & $659.67 \mathrm{E}$ & 77.92 & $43.57 \mathrm{GH}$ & 2.87 \\
\hline 12 & 7476 & 365.33BCD & 52.79 & 6.50DEF & 0.62 & $645.67 \mathrm{E}$ & 74.33 & $42.67 \mathrm{GH}$ & 3.45 \\
\hline 13 & 7479 & $368.00 \mathrm{BCD}$ & 21.52 & $7.00 \mathrm{D}$ & 0.66 & $832.67 \mathrm{D}$ & 81.75 & 46.53EFGH & 5.26 \\
\hline 14 & 7483 & $289.00 \mathrm{FG}$ & 12.00 & $8.87 \mathrm{BC}$ & 0.35 & $838.67 \mathrm{D}$ & 63.34 & $45.87 \mathrm{FGH}$ & 2.60 \\
\hline 15 & 7458 & $383.67 \mathrm{BC}$ & 10.26 & $6.07 \mathrm{EFG}$ & 0.15 & $841.33 \mathrm{D}$ & 64.03 & $50.40 \mathrm{CDEF}$ & 0.92 \\
\hline 16 & 7489 & $395.00 \mathrm{ABC}$ & 16.00 & $6.07 \mathrm{EFG}$ & 0.15 & $401.00 \mathrm{H}$ & 11.14 & $46.03 \mathrm{FGH}$ & 3.91 \\
\hline 17 & Giza 131 & $407.00 \mathrm{AB}$ & 14.18 & $9.23 \mathrm{AB}$ & 0.61 & $970.67 \mathrm{ABC}$ & 114.74 & $57.70 \mathrm{~A}$ & 4.13 \\
\hline 18 & Giza 2000 & $443.67 \mathrm{~A}$ & 41.48 & $9.97 \mathrm{~A}$ & 0.57 & $997.67 \mathrm{AB}$ & 105.5 & $58.97 \mathrm{~A}$ & 1.70 \\
\hline
\end{tabular}

\section{Weight of 1000 grain (g):}

It was calculated and data are given in table ( 7 and 8). Means ranged from 40.57 to $57.43 \mathrm{~g}$ for the genotype 7476 to cultivate Giza 2000, respectively, at the first season, and ranged from 41.9 to $58.97 \mathrm{~g}$ for the genotype 7466 to cultivate Giza 2000, respectively, at the second season.

Current study, detecting high genetic variation for the investigated morphological characters and grain yield, indicated that the barley landraces may be useful in selection and breeding programs. According to Marshall and Brown (1975) and Oka (1995), the numbers of genotypes (18) used in this study are sufficient to include all genetic variation under most circumstances.

Some promising genotypes were identified by using the variation within locally adapted barley germplasm.

Akgun et al. (2012) found that grain yield, was large for all populations and grain yield was 11.0-427.6 $\mathrm{g} \mathrm{m}-2$ among the landraces.

\section{Correlation between characters under study:}

In the first season of 2012/2013, the Length of the spike positive significant correlated with number of the days of shooting spikes (50\% spikes on plant). The negative significant correlations were between Green yield $(\mathrm{gm} / \mathrm{m} 2)$ and number of the days of shooting spikes ( $50 \%$ spikes on plant). Also, Number of the days of shooting spikes ( $50 \%$ spikes on plant) with Length of the spike was positive significant correlation Length of the spike and negative significant correlation with Green yield (gm/m2). (Table, 9)

In the second season of 2013/2014, Length of the spike positive significant correlation with number of the days of shooting spikes ( $50 \%$ spikes on plant). Also, the positive significant correlations were between numbers of the days of shooting spikes (50\% spikes on plant) with Length of the spike. Weight of 1000 grains (gm) was negative high significant correlation with Number of the days of shooting spikes ( $50 \%$ spikes on plant) (Table, 10)

The data obtained from this work revealed that seasons were found to be different, giving strong evidence that environmental factor affecting the tested characters 
Table 9. Correlation between characters under study during the first season of 2012/2013

\begin{tabular}{lcccccc}
\hline Characters & $\begin{array}{c}\text { Plant } \\
\text { height } \\
\text { (cm) }\end{array}$ & $\begin{array}{c}\text { Spike } \\
\text { length } \\
\text { (cm) }\end{array}$ & $\begin{array}{c}\text { Grain } \\
\text { yield } \\
\text { (g m-2) }\end{array}$ & $\begin{array}{c}\text { Number of days } \\
\text { to heading } \\
\text { (days) }\end{array}$ & $\begin{array}{c}\text { Number of } \\
\text { plant per } \\
\text { m2 }\end{array}$ & $\begin{array}{c}\text { Weight of 1000 } \\
\text { grain (grams) }\end{array}$ \\
\hline Plant height $(\mathrm{cm})$ & - & 0.216 & 0.202 & 0.198 & -0.196 & -0.083 \\
\hline Spike length $(\mathrm{cm})$ & & - & 0.091 & $0.277^{*}$ & 0.130 & 0.166 \\
\hline Grain yield(g m-2) & & & - & $-0.328^{*}$ & -0.210 & 0.054 \\
\hline $\begin{array}{l}\text { Number of days to } \\
\text { heading (days) }\end{array}$ & & & - & -0.192 & $-0.319^{*}$ \\
\hline Number of plant per m2 & & & & - & 0.197 \\
\hline $\begin{array}{l}\text { Weight of 1000 grain } \\
\text { (grams) }\end{array}$ & & & & & - \\
* correlation is singnificant at the 0.05 level (2-tailed). \\
** Correlation is singnificant at the 0.01 level (2-tailed).
\end{tabular}

Table 10. Correlation between characters under study during the second season of 2013/2014

\begin{tabular}{|c|c|c|c|c|c|c|}
\hline Characters & $\begin{array}{c}\text { Plant } \\
\text { height } \\
\text { (cm) }\end{array}$ & $\begin{array}{l}\text { Spike } \\
\text { length } \\
\text { (cm) }\end{array}$ & $\begin{array}{c}\text { Grain } \\
\text { yield } \\
\text { (g m-2) }\end{array}$ & $\begin{array}{c}\text { Number of } \\
\text { days to heading } \\
\text { (days) }\end{array}$ & $\begin{array}{r}\text { Number of } \\
\text { plant per } m 2\end{array}$ & $\begin{array}{l}\text { Weight of } 1000 \\
\text { grain (grams) }\end{array}$ \\
\hline Plant height $(\mathrm{cm})$ & - & 0.239 & 0.096 & 0.215 & -0.257 & $0 . .037$ \\
\hline Spike length $(\mathrm{cm})$ & & - & 0.013 & $0.269 *$ & 0.127 & 0.185 \\
\hline Grain yield(g m-2) & & & - & -0.188 & -0.262 & 0.041 \\
\hline $\begin{array}{l}\text { Number of days to heading } \\
\text { (days) }\end{array}$ & & & & - & -0.136 & $-0.382 * *$ \\
\hline Number of plant per m2 & & & & & - & 0.118 \\
\hline $\begin{array}{l}\text { Weight of } 1000 \text { grain } \\
\text { (grams) }\end{array}$ & & & & & & - \\
\hline
\end{tabular}

\section{REFERENCES}

Akar, T., M. Avci and F. Dusunceli 2012. Post-harvest operations. Food and Agriculture Organization of the United Nations.1-64.

http://www.fao.org/3/a-au997e

Akgun, N., A. Topal and M. Akcura 2012. Evaluation of central Anatolian barley landraces. Agric. Res., 29: 87-93.

Akimaliev, D. 2006. Assessment of the modern condition and development of strategies of plant breeding and biotechnology in Central Asia.

Marshal, D. R. and Brown, A. H. D., 1975. Optimum sampling strategies in genetic conservation. In: $\mathrm{OH}$ Frankel and JG Hawkes (Eds), Crop Genetic Resources for Today and Tomorrow: 53-80, Cambridge University Press, Cambridge.

Morrell, P. L. and, M. T. Clegg 2007. Genetic evidence for a second domestication of barley (Hordeum vulgare) east of the Fertile Crescent. Proceedings of the National Academy of Sciences 104(9), 3289-3294.
Nevo, E. (1992). Origin, evolution, population genetics and resources for breeding of wild barley, Hordeum spontaneum in the fertile crescent. In Barley: Genetics, Biochemistry, Molecular Biology and Biotechnology, Shewry, P. R., Ed., CAB International: Wallingford, UK, pp. 19-43.

Oka, H. I., 1995. Consideration on the population size necessary for conservation of crops germplasms. In: T. Matuso (ed.), Japan International Biological ProgramJIBP Synthesis, 5, Tokyo: 57-63.

Ullrich S. E. 2011. Significance, adaptation, production, and trade of barley. In Barley: Production, Improvement, and Uses. Wiley Blackwell, pp.3-13.

Usubaliev, B., A. Brantestam, B. Salomon, L. GarkavaGustavsson and R. Bothmer 2013. Genetic diversity in farmer grown spring barley material from Kyrgyzstan. Genetic Resources and Crop Evolution, 1-16.

Weissteiner C. J. and W. Kühbauch 2005. Regional yield forecasts of malting barley (Hordeum vulgare L.) by NOAA-AVHRR remote sensing data and ancillary data. Journal of Agronomy and Crop Science, 191: 308-320. 
Wright, S. 1978. Evolution and the genetics of populations. Variability within and among natural populations. Vol. 4. University of Chicago press, Chicago, IL, USA

Zohary, D. and M. Hopf 1988. Domestication of plants in the Old World. The origin and spread of cultivated plants in West Asia, Europe and the Nile Valley: Clarendon Press. ISBN 0198541988.
Zhao C., L. Liu, J. Wang, W. Huang, X. Song and C. Li 2005. Predicting grain protein content of winter wheat using remote sensing data based on nitrogen status and water stress. International Journal of Applied Earth Observation and Geoinfo, 7: 1-9

\section{الملغص الهرب - | (المي \\ قيم بهض الأصط الورالثية للثعير المعبر \\ محمد عبد العزيزمحمد علمر وخمسيس إبراهيمسعد}

الاصول الوراثية سا، 0 وسّا كلتت اعلى الاصول

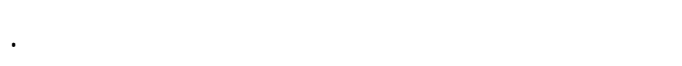

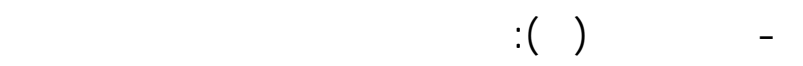
الاصول الوراثية في كلا المونمين.

0

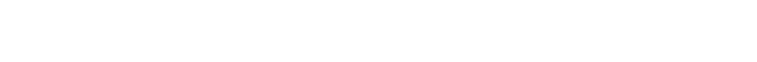

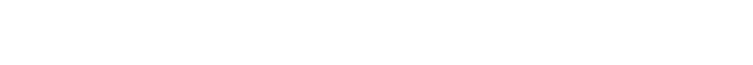
أعلى من الاصول الوراثية الاخرى.

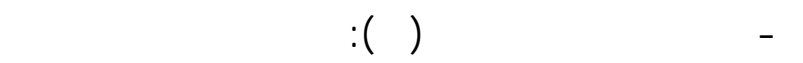

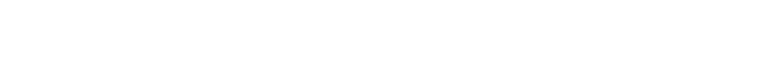

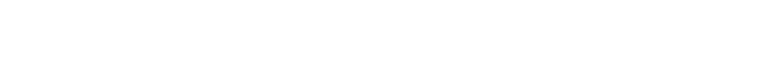

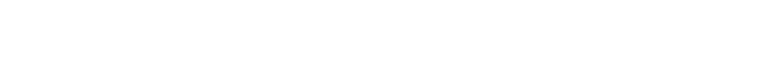

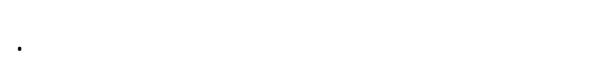

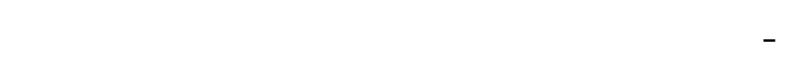

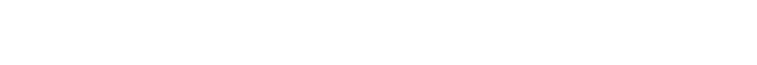
سلبى ومعنوى مع صفة المحصول لكن صفة ل...

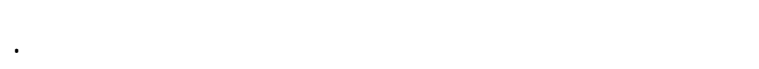

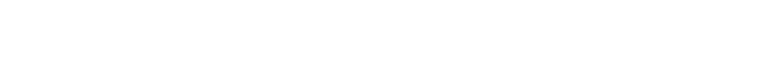

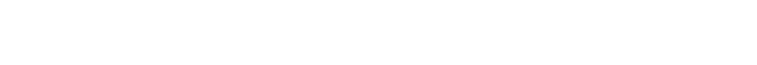
وكلن عالى المعنوية.
قم تقيذ هذا البهث لقييم 1/ تركيب ورالث منهم 17

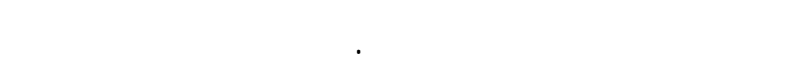

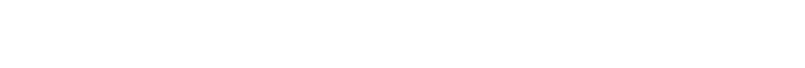

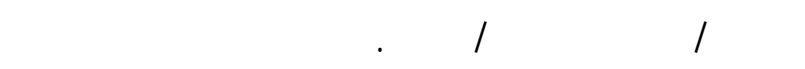

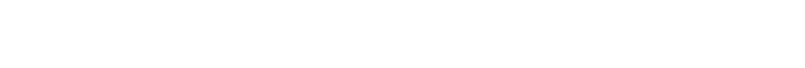
ويتضح من النتائج قفوق الأصنا النف النجارية على الأصول الوراثية وتلاظظ الآتى:

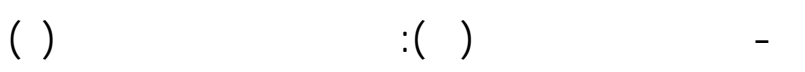

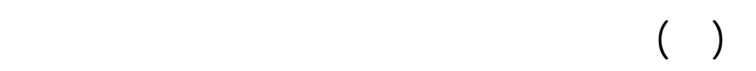

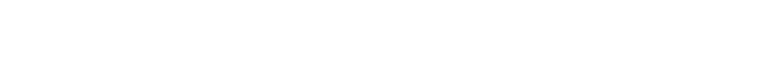

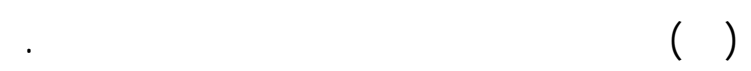

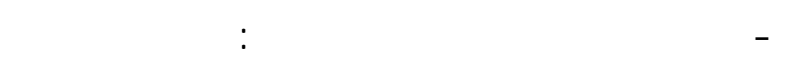

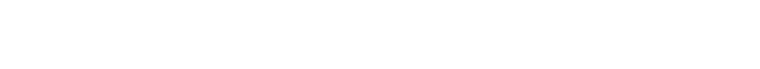
الاصنف التجارية وباقى الاصول الوراثية وفى العلم

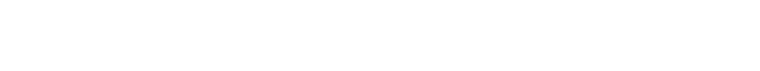

$$
\text { مقارنة بالاصنف التجارية. }
$$

ب - مفة محصول الحبوب: فى المونم الاول الاصول

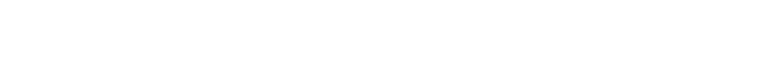

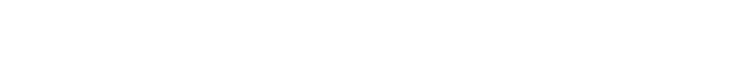

